\title{
Development of Guidelines on the Primary Prevention of Frailty in Community-Dwelling Older Adults
}

\author{
Seungkook Ki ${ }^{1,2}$, Ji-hye Yun ${ }^{1,3}$, Yunhwan Lee ${ }^{1,3}$, Chang-Won Won ${ }^{4}$, Miji Kim ${ }^{5}$, Chang-O Kim ${ }^{6}$, Ki Young Son ${ }^{7}$, Hyuntae Park $^{8}$, \\ Saejong Park ${ }^{9}$, Kyung-Eun Lee ${ }^{10}$, Sang Joon Son ${ }^{11}$, Kirang Kim ${ }^{12}$, Mi Kyung Kim ${ }^{13}$, Jinhee Kim ${ }^{1,3}$ \\ ${ }^{1}$ Department of Preventive Medicine and Public Health, Ajou University School of Medicine, Suwon, Korea \\ ${ }^{2}$ Home Doctor Preventive Medicine Clinic, Suwon, Korea \\ ${ }^{3}$ Institute on Aging, Ajou University Medical Center, Suwon, Korea \\ ${ }^{4}$ Department of Family Medicine, Kyung Hee University, Seoul, Korea \\ ${ }^{5}$ Department of Biomedical Science and Technology, East-West Medical Research Institute, Kyung Hee University College of Medicine, Seoul, Korea \\ ${ }^{6}$ Institute of Social Welfare, Sungkonghoe University, Seoul, Korea \\ ${ }^{7}$ Department of Family Medicine, Asan Medical Center, University of Ulsan College of Medicine, Seoul, Korea \\ ${ }^{8}$ Department of Health Care Science, Dong-A University, Busan, Korea \\ ${ }^{9}$ Korea Institute of Sport Science, Seoul, Korea \\ ${ }^{10}$ Major of Food and Nutrition, Seoul Women's University, Seoul, Korea \\ ${ }^{11}$ Department of Psychiatry, Ajou University School of Medicine, Suwon, Korea \\ ${ }^{12}$ Department of Food Science and Nutrition, Dankook University, Cheonan, Korea \\ ${ }^{13}$ Department of Preventive Medicine, Hanyang University College of Medicine, Seoul, Korea
}

Corresponding Author:

Yunhwan Lee, MD, DrPH

Department of Preventive Medicine and Public Health, Ajou University

School of Medicine, 164 World cup-ro,

Yeongtong-gu, Suwon 16499, Korea

E-mail: yhlee@ajou.ac.kr

ORCID:

https://orcid.org/0000-0001-8484-4750

Received: July 1, 2021

Revised: November 10, 2021

Accepted: November 23, 2021
Background: Despite the increasing number of older adults as the population ages, there is a lack of frailty prevention guidelines for community-dwelling older adults. The Korean Frailty and Aging Cohort Study conducted systematic review on contributors to frailty and developed guidelines on the primary prevention of frailty in community-dwelling older adults. Methods: This study updated a previous systematic review of contributors to frailty by adding the most recent articles. Based on this updated systematic review, experts in geriatrics and gerontology developed guidelines for preventing frailty using the Delphi method. Results: These guidelines categorized the recommendations into physical activity, resilience, oral health, management of non-communicable diseases, involvement in society, smoking cessation, and eating various kinds of food. Conclusion: Unlike previous frailty-related guidelines, this study developed evidence-based frailty prevention guidelines based on a systematic review. The guidelines are expected to contribute to the healthy aging of community-dwelling older adults by the primary prevention of frailty.

Key Words: Frailty, Primary prevention, Guideline, Aged, Independent living

\section{INTRODUCTION}

The aging population is a global trend. The proportion of the world's population aged 65 years or older is projected to reach $15.9 \%$ of the total population by 2050 . $^{1)}$ The results of several studies have shifted the perspective of aging from chronological to biological age, resulting in the concept of frailty. ${ }^{2)}$

Frailty is a vulnerable condition in which the intrinsic capacity to respond to internal and external stressors is weakened, increasing the likelihood of adverse health outcomes such as disability, hospitalization, institutionalization, and death. ${ }^{3-6)}$ Eventually, frailty is a state of declined biological homeostasis that resists entropy. ${ }^{2)}$

The prevalence of frailty is global. The reported community prevalence in high-income countries is $10 \%-12 \%{ }^{7,8)}$ and increases with age to $15.7 \%$ and $26.1 \%$ in those aged $80-84$ and 85 years or 
older, respectively. ${ }^{7)}$ The prevalence of prefrailty is reported at $41.6 \%$. Therefore more than half of the older population in high-income countries is related to frailty or prefrailty. The prevalence of frailty and prefrailty in middle-and low-income countries is $17.4 \%$ and $49.3 \%$, respectively, higher than that in high-income countries. ${ }^{9)}$ In Korea, the reported prevalence of frailty and prefrailty is $2.5 \%-12.4 \%$ and $27.7 \%-49.7 \%$, respectively. ${ }^{10)}$

A recent meta-analysis reported an incidence of frailty and prefrailty in communities of 43.4 and 150.6 cases per 1,000 person-years, respectively. The change from robust to frailty was 12.0 cases per 1,000 person-years, whereas the change from prefrailty to frailty was 62.7 cases per 1,000 person-years. ${ }^{11)}$

The critical point in frailty is that it is a dynamic concept that frailty is preventable and can be reversed to robustness in some cases. ${ }^{12-14)}$ Nevertheless, there remains a lack of frailty prevention guidelines for community-dwelling older adults. Moreover, despite the importance of self-care in frailty prevention, literature reviews of modifiable contributors to frailty are also lacking.

Therefore, this study conducted a systematic review on contributors to frailty and applied the Delphi method to develop frailty prevention guidelines for community-dwelling older adults. These evidence-based guidelines can contribute to the primary prevention of frailty by improving self-care among community-dwelling older adults.

\section{MATERIALS AND METHODS}

\section{Purpose and Scope}

The purpose of these guidelines is to prevent frailty by helping community-dwelling older adults avoid frailty risk factors and obtain/maintain protective factors. In this context, this study developed evidence-based guidelines with which older adults can comply in the real world (Table 1).

\section{Team}

The guidelines were developed as part of the Korean Frailty and Aging Cohort Study (KFACS). The KFACS is a nationwide multicenter study that aimed to identify and analyze causes and effects of aging. ${ }^{15,16)}$ The guideline development team comprised a multidisciplinary group, including three geriatricians, three preventive medicine and public health experts, four nutritionists, three physical education experts, and one social welfare expert.

\section{Key Questions}

The detailed contents of structured key questions for the article search are shown in Table 2. Since there has not been a clear summary of the contributors of frailty previously, the intervention/exposure items have been comprehensively set to cover as many contributors as possible. The outcomes were limited to physical frailty identified by validated tools based on the phenotype or cumulative deficit model. In addition, the study design was limited to cohort studies considering that randomized clinical trials that set outcomes as the occurrence of frailty are challenging to conduct and recommendations should be applicable to the community.

\section{Evidence Retrieval}

The search strategy, including systematic reviews, was based on a decision tree of the systematic review in the WHO Handbook for

Table 1. Scope of developing the guideline

\begin{tabular}{ll}
\hline PIPOH & \multicolumn{1}{c}{ Scope } \\
\hline $\begin{array}{l}\text { Population } \\
\text { Intervention }\end{array}$ & $\begin{array}{l}\text { Community-dwelling adults aged } 60 \text { years or older } \\
\text { Avoidance of risk factors of frailty } \\
\text { Acquisition/maintenance of factors protective against } \\
\text { frailty }\end{array}$ \\
Professionals/patients & Community-dwelling adults aged 60 or older \\
Outcome & Physical frailty \\
Healthcare setting & Community
\end{tabular}

Table 2. PICOTSS framework for key questions

\begin{tabular}{ll}
\hline PICOTSS & Contents \\
\hline $\begin{array}{l}\text { Population } \\
\text { Intervention/exposure }\end{array}$ & $\begin{array}{l}\text { Modifiable contributors (physical activity, nutrition, cognitive activity, social activity, and other lifestyles), management of non-commu- } \\
\text { nicable diseases }\end{array}$ \\
Comparison & $\begin{array}{l}\text { Agroup not acquiring/maintaining modifiable protective factors } \\
\text { Agroup not avoiding modifiable risk factors }\end{array}$ \\
Outcome & $\begin{array}{l}\text { Agroup not managing non-communicable diseases } \\
\text { Physical frailty }\end{array}$ \\
Setting & A follow-up period of 1 year or more \\
Study design & Community \\
\hline
\end{tabular}


Guideline Development. ${ }^{17)}$ First, a systematic review published in 2017 was identified as a result of searching for systematic reviews related to the key questions. ${ }^{18)}$ The quality of this systematic review was evaluated by assessing the methodological quality of systematic reviews (AMSTAR 2) ${ }^{19)}$ It was evaluated to be of high quality. Therefore, the development team used it for guideline development.

However, since this systematic review was conducted on articles published between January 2005 and September 2016, the development team updated the systematic review by adding more recent articles (January 2016-December 2018). To minimize missing articles, the overlapping period of 9 months was created.

For consistency, we used the same inclusion criteria as those used in a previous systematic review to search for updates. First, the included articles were as follows: (1) original articles, (2) articles related to contributors of frailty, (3) articles on community-dwelling older adults, and (4) articles whose outcome was frailty. Second, we included articles that used (1) a longitudinal study design, (2) a clear definition of frailty, and (3) specific tools to measure frailty and that included (4) subjects aged 60 years or older. The search formula was the same as that used in the previous systematic review. The search sources were the Embase on Ovid, Ovid MEDLINE, Cochrane Library, Web of Science, and CINAHL EBSCOhost databases.
Two public health experts (doctor 1, MPH 1) independently reviewed the titles and abstracts of the identified articles to select those that met the inclusion criteria. In the case of disagreement, the inclusion of an article was decided through discussion with a third party. Next, two experts independently conducted a full-text review of the selected articles, with disagreements resolved through discussion with a third party.

The quality of each article was also evaluated using the Quality of Reporting of Observational Longitudinal Research, as described in a previous systematic review. ${ }^{20)}$ The total score for this tool was 30 points. As in the previous systematic review, articles with $>15$ and $>20$ points were judged to be of adequate and high quality, respectively. Likewise, two people evaluated each article independently and resolved disagreements through discussion with a third party.

The articles included in the previous systematic review were also reviewed. In addition, articles unsuitable for developing guidelines, such as articles irrelevant to frailty prevention and those about contributors that could not be modified, were removed. Finally, 28 articles were selected for guideline development (Fig. 1). All selected articles used the phenotype model, which evaluates physical functions such as gait speed and handgrip strength but does not evaluate other areas such as cognition, social status, and oral health. The list of all articles can be found in Supplement A.

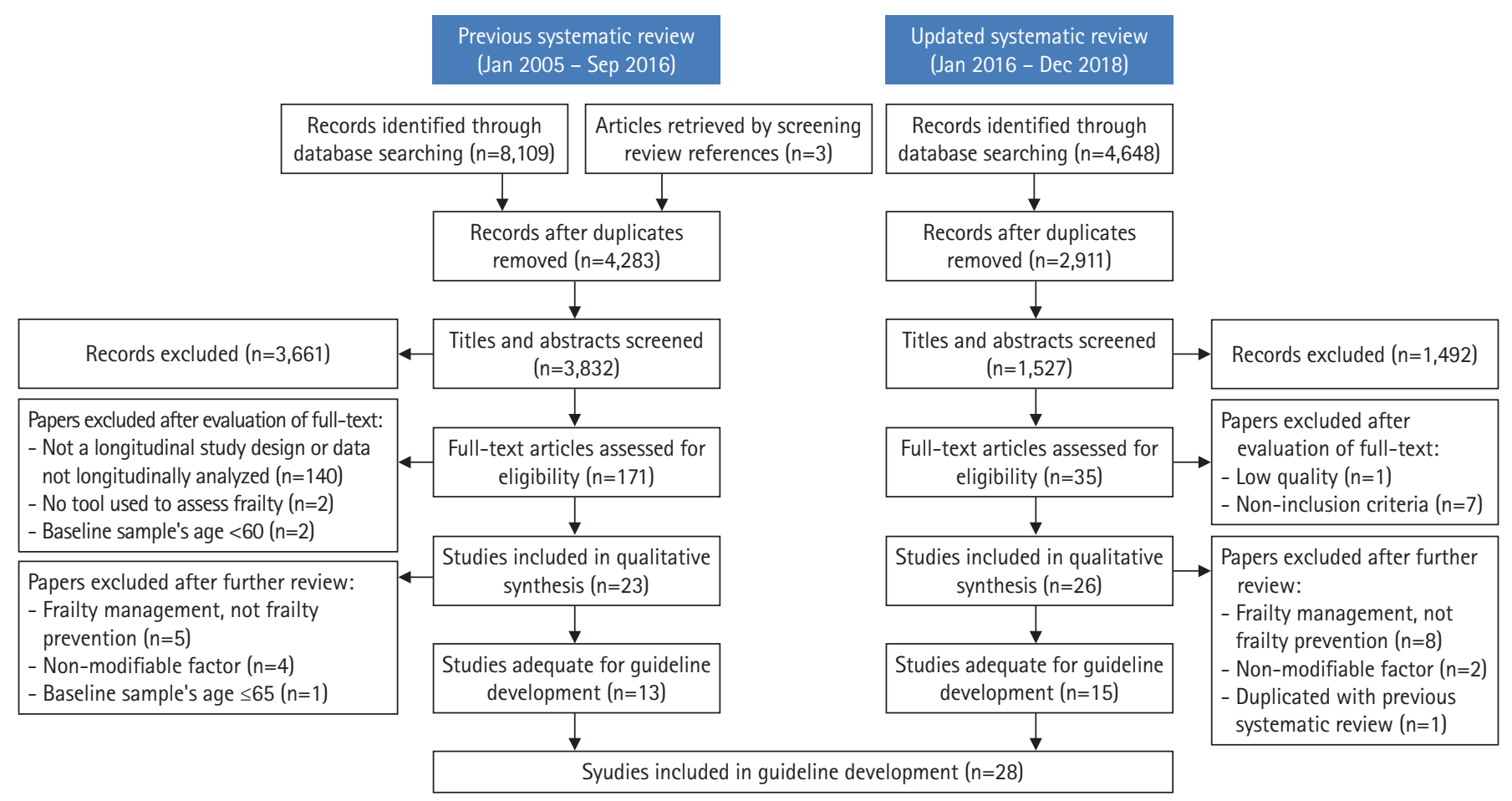

Fig. 1. The Preferred Reporting Items for Systematic Reviews and Meta-Analyses (PRISMA) flow chart. 


\section{Draft Guideline Statements}

From the 28 selected articles, 51 recommendations related to the prevention of frailty were prepared by organizing the contributors to frailty. However, the Grading of Recommendations Assessment, Development, and Evaluation (GRADE) system was not applied. The GRADE is a representative grading system to evaluate articles according to the certainty of the evidence and strength of the recommendations. The certainty of the evidence is rated based on the study design, while the strength of the recommendations is rated according to the certainty of the evidence, benefit-harm assessment, preference and value, and cost-effectiveness. ${ }^{21)}$

These guidelines are based on cohort studies; therefore, the certainty of the evidence was homogenous and low. In addition, these guidelines have little harm, high applicability, and high cost-effectiveness; thus, the strength of the recommendations is also likely to be homogeneous and strongly recommended.

However, given that the target population is community-dwelling older adults, the gap between low certainty of evidence and strong recommendations is likely to confuse the target population. Moreover, information beyond the guideline text itself is likely to impair the applicability to community-dwelling older adults. Consequently, there was little benefit in the application of the grading system.

\section{Reaching Agreement on Recommendations}

We applied the Delphi method to reach a consensus. A questionnaire was prepared for each recommendation, with answer sheets made on a 9-point Likert scale. The Delphi survey was conducted in two rounds. The second round included only recommendations that did not reach a consensus in the first round. Agreement was defined as $75 \%$ or more answers within 7-9 points, ambivalence as $75 \%$ or more answers within $4-6$ points, and disagreement as $75 \%$ or more answers were $1-3$ points. ${ }^{22,23)}$

Finally, a consensus was reached on 41 recommendations. Except for one recommendation, which reached ambivalence, all others reached an agreement. To improve the acceptability and usability among community-dwelling older adults, the recommendations are categorized into six domains: resilience, oral health, non-communicable disease management, involvement in society, smoking cessation, and eating various kinds of food.

However, no cohort study has assessed physical activity, which is a significant frailty intervention. As randomized clinical trials have evaluated physical activity, the guideline development team included physical activity recommendations from the Development of Clinical Intervention Guidelines on Prevention and Management of Frailty (unpublished) developed in the second year of the KFACS in these guidelines.

This unpublished guideline was developed by conducting a sys- tematic review (Supplement B) and then implementing the Delphi method. It recommended exercise and combined interventions of exercise and nutrition to improve frailty (Supplement C). However, only one of the supporting evidence in this unpublished guideline included non-frail subjects in the study and could be a study related to primary prevention. Therefore, these prevention guidelines recommended physical activity based on supporting evidence.

Through this process, the present guidelines for frailty prevention were established. After external review, these guidelines comprising seven domains, including physical activity, were finalized.

\section{RESULTS}

The guidelines for frailty prevention categorized the recommendations into seven domains: physical activity, resilience, oral health, management of non-communicable disease, involvement in society, smoking cessation, and eating various kinds of food. This study also created the "PROMISE" mnemonic to define and disseminate these guidelines (Table 3).

\section{Physical Activity}

\section{Make multicomponent exercise a way of life}

The prevalence of frailty is 1.9 times higher among those who do not perform multicomponent exercise than among those who do. Multicomponent exercise refers to physical activities that include resistance, aerobics, balance, and flexibility training. The intensity of these multicomponent exercises should be increased incrementally over 2-3 weeks. Multicomponent exercise can reduce sedentariness, prevent frailty, and even prevent disability. ${ }^{24)}$

\section{Resilience}

\section{Manage psychological problems with mental health professionals} A positive effect reduced the risk of frailty by $8 \%{ }^{25)}$ Additionally, the higher the number of depressive symptoms, the greater the risk of frailty, ${ }^{26)}$ which increases by up to 2.2 fold. ${ }^{27)}$ Moreover, ap-

Table 3. Frailty prevention mnemonics: PROMISE

PROMISE mnemonics
Physical activity
Resilience
Oral health
Management of non-communicable diseases
Involvement in society
Smoking cessation
Eating various kinds of food


athy and high levels of loneliness increase frailty risk by 2.9 and 1.9 times, respectively. ${ }^{28,29)}$ Therefore, psychological difficulties in old age should be managed with the help of mental health professionals.

\section{Oral Health}

\section{Take care of your teeth and gums}

Each additional tooth preserved in old age reduces the risk of frailty by $5 \%{ }^{30)}$ A weak maximum bite force increases the risk of frailty by $2.8 \mathrm{fold}^{31)}$ and severe periodontitis increases the risk of frailty by 2.1 fold. Teeth should also be brushed three times daily to maintain oral hygiene, while dentures should be washed daily. Finally, oral health checkups and tartar removal should be performed every 6 months to manage oral health.

\section{Management of Non-communicable Diseases}

\section{Actively manage your chronic conditions}

High blood pressure, ${ }^{27,30)}$ diabetes, stroke, chronic obstructive pulmonary disease, ${ }^{27)}$ osteoporosis, ${ }^{30)}$ metabolic syndrome, ${ }^{32)}$ arthritis $^{27)}$ and chronic pain ${ }^{33)}$ increase the risk of frailty. In old age, non-communicable diseases must be actively managed by regularly seeing a doctor. Vision and hearing impairment are additional health challenges that are easily overlooked. The risk of frailty increases by 2.1 fold when vision is impaired ${ }^{34)}$ and by 1.4 fold when hearing is impaired. ${ }^{35)}$ Problems with vision or hearing should be managed by a doctor.

\section{Review medications regularly}

The risk of frailty increases by 5.6 fold in people who take six or more drugs. ${ }^{36)}$ Older adults often take various drugs for multimorbidity. Regular medication reviews should be conducted to evaluate polypharmacy, and overlapping or unnecessary drugs should be discontinued.

\section{Involvement in Society}

\section{Meet people often}

Decreased social activities, social roles, or relationships (social frailty) increase the risk of frailty by 3.9 fold. ${ }^{37)}$ These guidelines recommend going out and visiting friends as often, in addition to talking to people every day, and if this is difficult, talking over the phone is recommended.

\section{Take care of yourself and your partner}

A spouse's depressive symptoms increase the risk of one's depres- sive symptoms; similarly, a spouse's frailty also increases an individual's risk of frailty. ${ }^{38)}$ Therefore, frailty prevention with a partner is more effective than alone.

\section{Smoking Cessation}

\section{Stop smoking}

Smoking increases the risk of frailty by $1.5-2.9$ times. ${ }^{26,27,39)}$ Smoking cessation positively affects health, even in old age. Smoking causes various diseases and frailty; therefore, we recommend quitting smoking as soon as possible.

\section{Eating Various Kinds of Food}

\section{Maintain a balanced diet}

The consumption of fish, ${ }^{40)}$ fruits and vegetables, ${ }^{41)}$ low-fat milk, and low-fat yogurt ${ }^{42)}$ reduces the risk of frailty. In old age, eating habits are fixed, and preparing meals is challenging; therefore, older adults frequently eat simple meals such as rice, kimchi, or instant foods. These unbalanced diets increase the risk of frailty; therefore, we recommend the conscious eating of a variety of foods.

\section{Eat sufficient food}

Lower intakes of protein and vitamin D increase the risk of frailty by up to 2.4 fold $^{43)}$ and 1.6 fold ${ }^{26)}$ respectively. Intakes of vitamin B6, folic acid, vitamin $\mathrm{C}$, and vitamin $\mathrm{E}$ below the recommended dietary allowances (RDA) also increase the risk of frailty (Supplement D) ${ }^{44)}$ Among the 10 vitamins, older adults with $<5$ RDAs have a 2.8 -fold increased risk of frailty. ${ }^{44)}$ In addition, being underweight (body mass index $<18.5 \mathrm{~kg} / \mathrm{m}^{2}$ ) increases the risk of frailty by 1.7 fold. ${ }^{27)}$ Therefore, eating can prevent frailty. However, as the risk of frailty increases by 1.4-4 fold in older adults with or at risk of obesity (body mass index $\geq 30 \mathrm{~kg} / \mathrm{m}^{2}$ ), ${ }^{26,27)}$ food consumption should be reduced.

\section{DISCUSSION}

This study developed guidelines for the primary prevention of frailty in community-dwelling older adults based on the results of a systematic review. Because older adults have complex medical conditions owing to physiological changes, multimorbidity, polypharmacy, and interactions, ${ }^{45)}$ the approach to frailty should be comprehensive. ${ }^{46)}$ By setting comprehensive key questions for the systematic review, the development team tried to capture the contributors of frailty as much as possible.

Based on the identified evidence, experts in geriatrics and gerontology derived recommendations using the Delphi method. As the 
users of these guidelines are community-dwelling older adults and not health practitioners, this study attempted to improve the acceptability of the guidelines by organizing recommendations spanning various areas. Thus, we categorized the guidelines for preventing aging into seven areas: physical activity, resilience, oral health, chronic disease management, social participation, smoking cessation, and various food intake.

However, this study had some limitations. First, the evidence used to develop these guidelines was based on the results of cohort studies. Thus, the recommendations lacked certainty of the evidence and it was not easy to apply the GRADE system to the guidelines. Second, because the guidelines were developed based on known contributors to frailty, the recommendations were inevitably presented in a limited area.

In conclusion, these frailty prevention guidelines were developed by reflecting the contributors to frailty through a systematic review. The guidelines must be continuously updated by considering new scientific evidence. Therefore, the development team plans to update these guidelines through regular systematic reviews and also develop frailty management guidelines for health practitioners.

We hope that these guidelines based on scientific evidence will contribute to the prevention of frailty and help the healthy aging of community-dwelling older adults. A Korean version of the guideline is provided in Supplement E.

\section{SUPPLEMENTARY MATERIALS}

Supplementary materials can be found via https://doi.org/10. 4235/agmr.21.0072.

\section{ACKNOWLEDGMENTS}

\section{CONFLICT OF INTEREST}

The researchers claim no conflicts of interest.

\section{FUNDING}

This work was supported by the Korea Health Technology R\&D Project through the Korea Health Industry Development Institute (KHIDI), Ministry of Health and Welfare, Republic of Korea (No. HI15C3153).

\section{AUTHOR CONTRIBUTION}

Conceptualization, SK, JY, YL, CWW; Data curation, SKK, JHY; Funding acquisition, YL, CWW; Investigation, SK, JY; Methodology, SK, JY, JK; Project administration, SK, YL, JK; Resources, MK, COK, KYS, HP, SP. KEL, SJS, KK, MKK; Supervision, YL,
CWW, MK, COK, KYS, HP, SP. KEL, SJS, KK, MKK, JK; Writing-original draft, SK; Writing-review \& editing, SK, JY, YL, JK.

\section{REFERENCES}

1. United Nations; Department of Economic and Social Affairs. World Population Prospects 2019: methodology of the United Nations population estimates and projections. New York, NY: United Nations; 2019.

2. Cesari M, Theou O. Frailty: the broad view. In: Fillit HM, Rockwood K, Young JB, editors. Brocklehurst's textbook of geriatric medicine and gerontology. Philadelphia, PA: Elsevier; 2017. p. 82-7.

3. Fried LP, Tangen CM, Walston J, Newman AB, Hirsch C, Gottdiener J, et al. Frailty in older adults: evidence for a phenotype.J Gerontol A Biol Sci Med Sci 2001;56:M146-56.

4. Rockwood K, Song X, MacKnight C, Bergman H, Hogan DB, McDowell I, et al. A global clinical measure of fitness and frailty in elderly people. CMAJ 2005;173:489-95.

5. Morley JE, Vellas B, van Kan GA, Anker SD, Bauer JM, Bernabei $\mathrm{R}$, et al. Frailty consensus: a call to action. J Am Med Dir Assoc 2013;14:392-7.

6. Clegg A, Young J, Iliffe S, Rikkert MO, Rockwood K. Frailty in elderly people. Lancet 2013;381:752-62.

7. Collard RM, Boter H, Schoevers RA, Oude Voshaar RC. Prevalence of frailty in community-dwelling older persons: a systematic review.J Am Geriatr Soc 2012;60:1487-92.

8. O'Caoimh R, Galluzzo L, Rodriguez-Laso A, Van der Heyden J, Ranhoff AH, Lamprini-Koula M, et al. Prevalence of frailty at population level in European ADVANTAGE Joint Action Member States: a systematic review and meta-analysis. Ann Ist Super Sanita 2018;54:226-38.

9. Siriwardhana DD, Hardoon S, Rait G, Weerasinghe MC, Walters KR. Prevalence of frailty and prefrailty among community-dwelling older adults in low-income and middle-income countries: a systematic review and meta-analysis. BMJ Open 2018;8:e018195.

10. Kim KJ, Shin J, Choi J, Won CW. Discrepancies in the prevalence of known frailty scales: Korean Frailty and Aging Cohort Study. Ann Geriatr Med Res 2018;22:137-44.

11. Ofori-Asenso R, Chin KL, Mazidi M, Zomer E, Ilomaki J, Zullo AR, et al. Global incidence of frailty and prefrailty among community-dwelling older adults: a systematic review and meta-analysis. JAMA Netw Open 2019;2:e198398.

12. Gill TM, Gahbauer EA, Allore HG, Han L. Transitions between frailty states among community-living older persons. Arch Intern Med 2006; 166:418-23. 
13. Ottenbacher KJ, Graham JE, Al Snih S, Raji M, Samper-Ternent R, Ostir GV, et al. Mexican Americans and frailty: findings from the Hispanic established populations epidemiologic studies of the elderly. Am J Public Health 2009;99:673-9.

14. Ferrucci L, Fabbri E, Walston JD. Frailty. In: Halter JB, Ouslander JG, Studenski S, editors. Hazzard's geriatric medicine and gerontology. 7th ed. New York, NY: McGraw-Hill; 2017.

15. Won CW, Lee S, Kim J, Chon D, Kim S, Kim CO, et al. Korean frailty and aging cohort study (KFACS): cohort profile. BMJ Open 2020;10:e035573.

16. Won CW, Lee Y, Choi J, Kim KW, Park Y, Park H, et al. Starting construction of frailty cohort for elderly and intervention study. Ann Geriatr Med Res 2016;20:114-7.

17. World Health Organization. WHO handbook for guideline development. 2nd ed. Geneva, Switzerland: World Health Organization; 2014.

18. Feng Z, Lugtenberg M, Franse C, Fang X, Hu S, Jin C, et al. Risk factors and protective factors associated with incident or increase of frailty among community-dwelling older adults: a systematic review oflongitudinal studies. PLoS One 2017;12:e0178383.

19. Shea BJ, Reeves BC, Wells G, Thuku M, Hamel C, Moran J, et al. AMSTAR 2: a critical appraisal tool for systematic reviews that include randomised or non-randomised studies of healthcare interventions, or both. BMJ 2017;358:j4008.

20. Tooth L, Ware R, Bain C, Purdie DM, Dobson A. Quality of reporting of observational longitudinal research. Am J Epidemiol 2005; 161:280-8.

21. Guyatt GH, Oxman AD, Vist GE, Kunz R, Falck-Ytter Y, Alonso-Coello P, et al. GRADE: an emerging consensus on rating quality of evidence and strength of recommendations. BMJ 2008;336:924-6.

22. Ministry of Health \& Welfare; Korean Association of Orthopedic Medicine. Adaptation process for developing Korean clinical practice guidelines. Seoul, Korea: Ministry of Health \& Welfare; 2011.

23. Diamond IR, Grant RC, Feldman BM, Pencharz PB, Ling SC, Moore AM, et al. Defining consensus: a systematic review recommends methodologic criteria for reporting of Delphi studies. J Clin Epidemiol 2014;67:401-9.

24. Cesari M, Vellas B, Hsu FC, Newman AB, Doss H, King AC, et al. A physical activity intervention to treat the frailty syndrome in older persons-results from the LIFE-P study. J Gerontol A Biol Sci Med Sci 2015;70:216-22.

25. Aranda MP, Ray LA, Snih SA, Ottenbacher KJ, Markides KS. The protective effect of neighborhood composition on increasing frailty among older Mexican Americans: a barrio advantage? J Aging Health 2011;23:1189-217.
26. Hoogendijk EO, van Hout HP, Heymans MW, van der Horst HE, Frijters DH, Broese van Groenou MI, et al. Explaining the association between educational level and frailty in older adults: results from a 13-year longitudinal study in the Netherlands. Ann Epidemiol 2014;24:538-44.

27. Woods NF, LaCroix AZ, Gray SL, Aragaki A, Cochrane BB, Brunner RL, et al. Frailty: emergence and consequences in women aged 65 and older in the Women's Health Initiative Observational Study. J Am Geriatr Soc 2005;53:1321-30.

28. Ayers E, Shapiro M, Holtzer R, Barzilai N, Milman S, Verghese J. Symptoms of apathy independently predict incident frailty and disability in community-dwelling older adults. J Clin Psychiatry 2017;78:e529-36.

29. Gale CR, Westbury L, Cooper C. Social isolation and loneliness as risk factors for the progression of frailty: the English Longitudinal Study of Ageing. Age Ageing 2018;47:392-7.

30. Castrejon-Perez RC, Jimenez-Corona A, Bernabe E, Villa-Romero AR, Arrive E, Dartigues JF, et al. Oral disease and 3-year incidence of frailty in mexican older adults. J Gerontol A Biol Sci Med Sci 2017;72:951-7.

31. Iwasaki M, Yoshihara A, Sato N, Sato M, Minagawa K, Shimada $\mathrm{M}$, et al. A 5-year longitudinal study of association of maximum bite force with development of frailty in community-dwelling older adults. J Oral Rehabil 2018;45:17-24.

32. Perez-Tasigchana RF, Leon-Munoz LM, Lopez-Garcia E, Gutierrez-Fisac JL, Laclaustra M, Rodriguez-Artalejo F, et al. Metabolic syndrome and insulin resistance are associated with frailty in older adults: a prospective cohort study. Age Ageing 2017;46: 807-12.

33. Megale RZ, Ferreira ML, Ferreira PH, Naganathan V, Cumming $\mathrm{R}$, Hirani V, et al. Association between pain and the frailty phenotype in older men: longitudinal results from the Concord Health and Ageing in Men Project (CHAMP). Age Ageing 2018;47:381-7.

34. Liljas AE, Carvalho LA, Papachristou E, De Oliveira C, Wannamethee SG, Ramsay SE, et al. Self-reported vision impairment and incident prefrailty and frailty in English community-dwelling older adults: findings from a 4-year follow-up study. J Epidemiol Community Health 2017;71:1053-8.

35. Liljas AE, Carvalho LA, Papachristou E, Oliveira C, Wannamethee SG, Ramsay SE, et al. Self-reported hearing impairment and incident frailty in English community-dwelling older adults: a 4-year follow-up study. J Am Geriatr Soc 2017;65:958-65.

36. Yuki A, Otsuka R, Tange C, Nishita Y, Tomida M, Ando F, et al. Polypharmacy is associated with frailty in Japanese community-dwelling older adults. Geriatr Gerontol Int 2018;18:1497500. 
37. Makizako H, Shimada H, Doi T, Tsutsumimoto K, Hotta R, Nakakubo S, et al. Social frailty leads to the development of physical frailty among physically non-frail adults: a four-year follow-up longitudinal cohort study. Int J Environ Res Public Health 2018; 15:490.

38. Monin J, Doyle M, Levy B, Schulz R, Fried T, Kershaw T. Spousal associations between frailty and depressive symptoms: longitudinal findings from the Cardiovascular Health Study. J Am Geriatr Soc 2016;64:824-30.

39. Kojima G, Iliffe S, Jivraj S, Liljas A, Walters K. Does current smoking predict future frailty?: the English longitudinal study of ageing. Age Ageing 2018;47:126-31.

40. Leon-Munoz LM, Guallar-Castillon P, Lopez-Garcia E, Rodriguez-Artalejo F. Mediterranean diet and risk of frailty in community-dwelling older adults. J Am Med Dir Assoc 2014;15:899903.

41. Garcia-Esquinas E, Rahi B, Peres K, Colpo M, Dartigues JF, Bandinelli S, et al. Consumption of fruit and vegetables and risk of frailty: a dose-response analysis of 3 prospective cohorts of community-dwelling older adults. Am J Clin Nutr 2016;104:
132-42.

42. Lana A, Rodriguez-Artalejo F, Lopez-Garcia E. Dairy consumption and risk of frailty in older adults: a prospective cohort study. J Am Geriatr Soc 2015;63:1852-60.

43. Sandoval-Insausti H, Perez-Tasigchana RF, Lopez-Garcia E, Garcia-Esquinas E, Rodriguez-Artalejo F, Guallar-Castillon P. Macronutrients intake and incident frailty in older adults: a prospective cohort study. J Gerontol A Biol Sci Med Sci 2016;71:132934.

44. Balboa-Castillo T, Struijk EA, Lopez-Garcia E, Banegas JR, Rodriguez-Artalejo F, Guallar-Castillon P. Low vitamin intake is associated with risk of frailty in older adults. Age Ageing 2018; 47:872-9.

45. Ferrucci L, Orini S. Comprehensive geriatric assessment: an updated perspective. In: Pilotto A, Martin FC, editors. Comprehensive geriatric assessment. Cham, Switzerland: Springer International Publishing; 2017.

46. Won CW. Frailty: its scope and implications for geriatricians. Ann Geriatr Med Res 2019;23:95-7. 